\title{
MAGEA10 expression is a predictive marker of early hepatic recurrence after curative gastrectomy for gastric and gastroesophageal junction cancer
}

\author{
Keiichi Fujiya ${ }^{1,2} \cdot$ Masanori Terashima $^{1}$ - Keiichi Ohshima ${ }^{3} \cdot$ Daisuke Aizawa $^{4} \cdot$ Takashi Sugino $^{4} \cdot$ \\ Masakuni Serizawa ${ }^{5} \cdot$ Kenichi Nakamura $^{1} \cdot$ Takeshi Nagashima $^{6,7} \cdot$ Keiichi Hatakeyama $^{3} \cdot$ Kenichi Urakami $^{6}$. \\ Yasuto Akiyama ${ }^{8} \cdot$ Yasuhiro Tsubosa $^{9} \cdot$ Yuko Kitagawa $^{2} \cdot$ Ken Yamaguchi $^{10}$
}

Received: 9 July 2020 / Accepted: 12 September 2020 / Published online: 23 September 2020

(c) The International Gastric Cancer Association and The Japanese Gastric Cancer Association 2020

\begin{abstract}
Background Resection for hepatic recurrence after gastrectomy in patients with gastric cancer may be curative; however, the prediction of hepatic recurrence remains intractable. Therefore, we aimed to explore predictive markers for hepatic recurrence in gastric and gastroesophageal junction cancer based on genetic information.

Methods This study recruited 154 patients who underwent curative gastrectomy for pathological stage II or III primary gastric and gastroesophageal junction adenocarcinoma. Genes associated with hepatic recurrence were comprehensively analyzed using whole-exome sequencing and gene expression profiling (GEP), followed by immunohistochemistry analysis for MAGEA10. The cumulative incidences of hepatic recurrence, relapse-free survival, and overall survival were evaluated. Results A total of 12 patients with early hepatic recurrences were found within 2 years of surgery. Although there were no distinct gene mutations in recurrent patients, upregulation of MAGEA10 was identified in patients with early hepatic recurrence using GEP analysis. Immunostaining for MAGEA10 stained the cell nuclei in 29 (18.8\%) of 154 samples. Furthermore, protein expression of MAGEA10 on immunohistochemistry was significantly related to a high MAGEA10 mRNA expression, high cumulative incidences of hepatic recurrence, and poor relapse-free survival. Overall survival did not differ significantly between positive and negative immunohistochemical staining for MAGEA10. The sensitivity and specificity of MAGEA10 staining for early hepatic recurrence were $58.3 \%$ and $84.5 \%$, respectively.

Conclusions MAGEA10 represents a promising predictive marker for early hepatic recurrence after curative gastrectomy for gastric and gastroesophageal junction cancer.
\end{abstract}

Keywords Gene expression profiling · Comprehensive analysis · MAGEA family

Electronic supplementary material The online version of this article (https://doi.org/10.1007/s10120-020-01123-y) contains supplementary material, which is available to authorized users.

Masanori Terashima

m.terashima@scchr.jp

1 Division of Gastric Surgery, Shizuoka Cancer Center, 1007 Shimonagakubo, Nagaizumi-cho, Sunto-gun, Shizuoka 411-8777, Japan

2 Department of Surgery, Keio University School of Medicine, Tokyo, Japan

3 Medical Genetics Division, Shizuoka Cancer Center Research Institute, Shizuoka, Japan

4 Division of Pathology, Shizuoka Cancer Center, Shizuoka, Japan
5 Drug Discovery and Development Division, Shizuoka Cancer Center Research Institute, Shizuoka, Japan

6 Cancer Diagnostics Research Division, Shizuoka Cancer Center Research Institute, Shizuoka, Japan

SRL, Inc., Tokyo, Japan

8 Immunotherapy Division, Shizuoka Cancer Center Research Institute, Shizuoka, Japan

9 Division of Esophageal Surgery, Shizuoka Cancer Center, Shizuoka, Japan

10 Shizuoka Cancer Center, Shizuoka, Japan 


\section{Introduction}

Recent progress in the perioperative treatment for advanced gastric cancer has contributed to an improvement in long-term survival [1]. However, the survival outcome after curative gastrectomy for more advanced gastric cancer remains unsatisfactory [2], and evidence that surveillance for detection of recurrence after gastrectomy improves survival is lacking [3]. Early detection of nonperitoneal recurrence may prolong survival as resection of the recurrent site is occasionally possible [4]; in particular, hepatectomies of liver metastases from gastric cancer may carry a survival benefit or provide a radical cure $[5,6]$. Moreover, survival after resecting a single metastasis is better than after resecting multiple metastases [7]. Hence, narrowing down patients at high risk of hepatic recurrence for detection at a relatively early stage is critical to improving survival outcomes.

Liver recurrence after gastrectomy cannot be precisely anticipated from tumor pathological factors, although it is known that liver metastasis with gastric cancer is frequently found in differentiated type adenocarcinoma [8]. A genetic approach has been used to estimate the risk of recurrence, and it is known that mutations in TP53 and PI3K/AKT pathway genes are more frequent in liver metastasis of gastric cancer than in non-metastatic gastric cancer $[9,10]$. A previous study used gene expression profiling (GEP) analysis of 63 preselected genes to demonstrate that TOP $2 A$ is associated with hematogenous recurrence [11]. However, the majority of previous studies that have targeted hepatic recurrence of gastric cancer only investigated a small number of genes or samples [9-14]. Therefore, because the statistical power to detect the genes associated with hepatic recurrence was lacking in previous studies, comprehensive analysis using whole-exome sequencing (WES) and GEP in large-scale samples is a promising future avenue of research.

This study aimed to explore predictive markers for early hepatic recurrence after curative gastrectomy using comprehensive analysis of genetic alterations and gene expression. Moreover, because MAGEA10 was detected in gene expression analysis, we investigated the efficacy of MAGEA10 as a promising predictive marker for early hepatic recurrence using immunohistochemistry.

\section{Materials and methods}

\section{Patients}

Between January 2014 and January 2017, surgically resected tumor specimens were obtained from 198 consecutive patients who underwent curative gastrectomy for pathological stage II or III primary gastric and gastroesophageal junction adenocarcinoma in Project HOPE (High-tech Omics-based Patient Evaluation) of Shizuoka Cancer Center [15]. Clinicopathological data were collected from patient records, and the tumor stage was assessed by the TNM 8th classification. Among the 198 patients, 44 patients were excluded for the following reasons: 9 patients with neoadjuvant chemotherapy, 1 patient with a low mRNA quality, 4 patients whose tumor mutation burden was below the lower limit of the normal range, and 30 patients with low tumor purity. As a result, 154 patients were included in the final analysis. To validate the result of immunohistochemistry for MAGEA10 in the other cohort, additional immunohistochemistry analysis was performed for 40 patients with early hepatic recurrence and 40 background-matched patients without recurrence, undergoing gastrectomy for gastric cancer between 2007 and 2017.

The research plan was designed according to the revised Ethical Guidelines for Human Genome/Gene Analysis Research in Japan. The Institutional Review Board of Shizuoka Cancer Center approved this study (approval number: 25-33 and 2019-25-2019-1-2). Written informed consent was obtained from all patients enrolled in this study. All experiments using clinical samples were carried out in accordance with the approved guidelines.

\section{Definition of recurrence}

Recurrences were diagnosed by imaging examination or pathological findings, and patterns of recurrence were categorized as hematogenous, peritoneal, distant lymph node, or locoregional recurrences. Early hepatic recurrence was defined as recurrence within 2 year of gastrectomy.

\section{Tissue samples}

Tumor tissue samples with sizes corresponding to weights of $\geq 100 \mathrm{mg}$ were dissected from resected specimens. Boardcertificated pathologists macroscopically dissected a tumorrich area and microscopically confirmed that the tissue sample included $\geq 50 \%$ tumor content.

\section{Whole-exome sequencing}

Detailed experimental protocols have been described previously [16-18]. WES was performed on an Ion Proton system (Thermo Fisher Scientific, Waltham, MA, USA) using Ion Ampliseq Exome kit (Thermo Fisher Scientific) according to the manufacturer's instructions. The exome library was constructed using an Ion Torrent AmpliSeq RDY Exome Kit (Thermo Fisher Scientific) [16, 17]. Pathogenic mutation 
was based on driver and likely driver mutations in the drivability assessment from multiple databases [18]. The WES data were applied to estimate the tumor purity using an in silico method, and low tumor purity was defined as $<20 \%$. Somatic copy number alterations (SCNA) were detected using Ion Reporter Software Copy Number Variation Analysis (Thermo Fisher Scientific). Amplification was defined as $\geq$ fivefold increase in gene expression and SCNA $\geq 4$, while deletion was defined as $\geq$ fivefold decrease in expression and $\mathrm{SCNA} \leq 1[18]$.

\section{Gene expression profiling}

GEP was performed using the same protocols as outlined previously $[18,19]$. Total RNA was extracted from approximately $10 \mathrm{mg}$ hashed tissue sample using the miRNeasy Mini Kit (Qiagen, Hilden, Germany) according to the manufacturer's instructions. A total of $100 \mathrm{ng}$ RNA was fluorescence labeled and hybridized to the SurePrint G3 Human Gene Expression $8 \times 60 \mathrm{~K}$ v2 Microarray (Agilent Technologies, Santa Clara, CA, USA), and data analysis was performed using the GeneSpring GX software program (Agilent Technologies). Samples with $>10$ in raw signal intensity values in tumor tissues were used. The fold change between tumor and normal mucosal tissues was calculated using the normalized intensity values. Differentially expressed genes (DEGs) were identified with a fold change of $>+4.0$ or $<-4.0$ and an FDR; a Benjamini-Hochberg procedure $p$ value of $<0.05$ was determined as the cut-off.

\section{Immunohistochemistry}

Resected specimens were fixed in $10 \%$ formalin, dehydrated, and embedded in paraffin. Samples were cut into 3- $\mu \mathrm{m}$ sections and mounted on glass slides. The sections were pretreated with epitope retrieval solution pH 9 (RE7119, Leica Microsystems Inc., Buffalo Grove, IL, USA) for $40 \mathrm{~min}$ at $95{ }^{\circ} \mathrm{C}$ and incubated at room temperature with primary anti-MAGEA10 rabbit polyclonal antibody at 1:200 dilution (PA5-50444, Thermo Fisher Scientific) overnight. Binding of the primary antibodies was detected using an EnVision + System-HRP Labelled Polymer (K4003, Agilent Technologies) and Liquid DAB + Substrate Chromogen System (K3468, Agilent Technologies). The slides were counterstained with hematoxylin. Positive controls were set to placenta. MAGEA10 protein expression by immunohistochemistry was evaluated by specialists in pathology (D.A. and T.S.) who were blinded to the clinicopathological data. Nuclear staining over $1 \%$ of the total was defined as positive staining. Staining in the cell cytoplasm only was judged as negative, since MAGEA10 is a nuclear protein [20].

\section{Statistical analyses}

Continuous variables are presented as the median and the first and third quartile. Statistical analyses were performed using the Fisher's exact test and Mann-Whitney $U$ test. Survival data were analyzed by the Kaplan-Meier method, and the log-rank test was used to compare groups. The cumulative incidence of recurrence was compared using the Fine-Gray regression model, and $p$ values and FDR $p$ values $<0.05$ were considered statistically significant. All statistical analyses were conducted using JMP version 12.2.0 (SAS Institute Inc., Cary, NC, USA) or R Statistics version 3.4.4 (R Foundation, Vienna, Austria).

\section{Results}

\section{Demographics of the study population}

The median total follow-up period was 1,202 days. Overall recurrence, hematogenous recurrence (including hepatic recurrence), peritoneal recurrence, lymph node recurrence, and locoregional recurrence were found in 46, 21, 18, 10, and 3 patients during follow-up, respectively. Among the 46 patients with overall recurrence, 34 (22.1\%) patients had early overall recurrence and 12 (7.8\%) of patients had early hepatic recurrence within 2 years of gastrectomy. Table 1 describes the clinicopathological differences according to early hepatic recurrence. There was no incidence of

Table 1 Clinicopathological characteristics according to early hepatic recurrence

\begin{tabular}{llll}
\hline & \multicolumn{2}{l}{ Early hepatic recurrence } & \multirow{2}{*}{$p$ value } \\
\cline { 2 - 3 } & Yes & No & \\
& $(n=12)(\%)$ & $(n=142)(\%)$ & 0.225 \\
\hline Age (years) & & & \\
$\quad$ Median & 69 & 70 & 0.743 \\
25th-75th percentile & $69-76$ & $66-79$ & \\
Sex & & & \\
Male & $8(67)$ & $102(72)$ & \\
Female & $4(33)$ & $40(28)$ & \\
Anatomical region & & & \\
Gastroesophageal junction & $2(17)$ & $18(13)$ & \\
Gastric & $10(83)$ & $124(87)$ & \\
Histology & & & \\
$\quad$ Differentiated type & $12(100)$ & $72(51)$ & \\
$\quad$ Undifferentiated type & 0 & $70(49)$ & \\
Pathological stage & & $56(39)$ & \\
II & $5(42)$ & $56(61)$ & \\
III & $7(58)$ & & \\
\hline
\end{tabular}


undifferentiated-type cancer in patients with early hepatic recurrence. Age, sex, anatomical region, and pathological cancer stage were not significantly different between patients with and without early hepatic recurrence.

\section{Gene mutation and early hepatic recurrence}

No gene mutations were significantly related to early hepatic recurrence by WES analysis. Figure 1 demonstrates a relationship between clinicopathological characteristics and the distribution of mutations, amplifications, and deletions in frequently detected oncogenes and cancer-related genes [18]. The mutated genes with high frequency $(>10 \%)$ were TP53 followed by MUC16, SYNE1, OBSCN, FAT3, KMT2D, $C S M D 3, L R P 1 B$, and PIK3CA. The proportion of major gastric cancer-specific gene mutations was similar to that in the data in The Cancer Genome Atlas and Japanese large genomic study [21, 22]. TP53 mutations were found in 71 (46.1\%) patients, of which 46 were missense mutations, 24 were truncating mutations, and 1 was an in-frame mutation. Among 71 patients with TP53 mutations, 62 (87.3\%) had pathogenic mutations, none of which were associated with early hepatic recurrence. Amplification was found in ERBB2

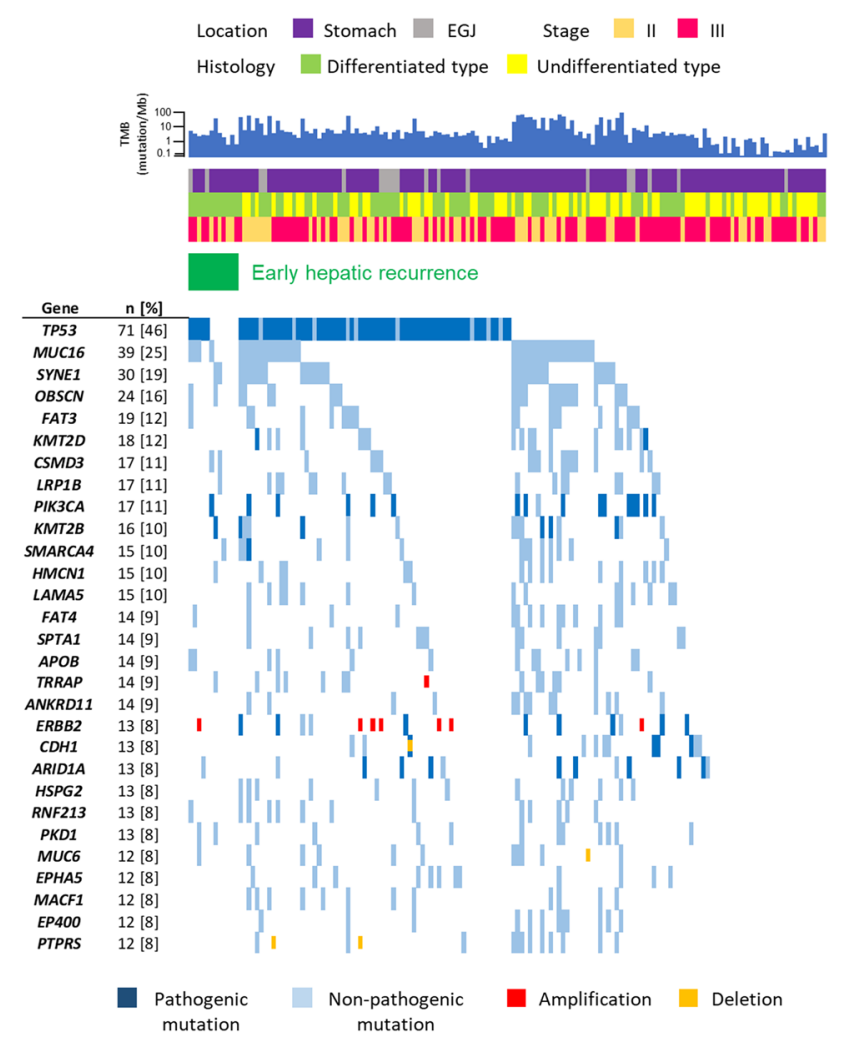

Fig. 1 Mutation, amplification, and deletion of the frequently detected oncogenes and cancer-related genes identified in 154 gastric and gastroesophageal junction cancer patients and TRRAP, while deletion was detected in PTPRS, CDH1, and MUC6.

\section{Identification of DEGs associated with early hepatic recurrence}

A volcano plot was used to visualize the DEGs between the presence and absence of all types of early recurrence (Fig. 2a). In tumors with overall early recurrence, 11 genes (SERPINA4, LYPD2, RXFP4, FGA, DUOX2, RDH12, THOC6, ORM2, SPINK1, GDF5, and CHIA) were significantly upregulated, while 2 genes (ASB5 and VSNL1) were significantly downregulated. It is considered that DEGs differ depending on the type of recurrence; therefore, a similar analysis was performed for DEGs in early hepatic recurrence. Figure $2 \mathrm{~b}$ demonstrates the DEGs in the presence or absence of early hepatic recurrence (Supplementary Table 1). Only MAGEA10 was significantly upregulated in tumors with early hepatic recurrence. Although no significant differences were observed, other MAGEA family genes, such as MAGEA1, MAGEA2B, MAGEA4, MAGEA6, MAGEA9, and MAGEA12, were also included in the 32 genes with DEGs with changes greater than fourfold. MAGEA family genes and 13 genes associated with overall early recurrence were not significantly different between the presence or absence of hematogenous, peritoneal, lymph node, or locoregional recurrence (Supplementary Table 2).

\section{Association with expression profiling of MAGEA10}

Figure 2c demonstrates high MAGEA10 mRNA expression in patients with early hepatic recurrence $(p=0.004)$. High MAGEA10 mRNA expression ( $\geq$ fourfold changes) was shown in 21 patients, including $6(50 \%)$ of the 12 patients with early hepatic recurrence, and 15 (10.6\%) of the 142 patients without early hepatic recurrence. MAGEA10 was barely expressed in normal tissue, while 6 of the 12 patients with early hepatic recurrence had low to moderate expression in tumor tissue (Fig. 2d). Figure 2e shows the expression pattern of MAGEA family genes. Among the 21 patients with high MAGEA10 mRNA expression, the coexpression of MAGEA10 with MAGEA2B, MAGEA12, MAGEA6, MAGEA4, and MAGEAl (in $90.5 \%$ of patients); MAGEA10 with MAGEA9 and MAGEA8 (in $81.0 \%$ of patients), and MAGEA10 with MAGEAIl (in $66.7 \%$ of patients) was detected.

\section{Immunohistochemistry for MAGEA10}

MAGEA10 mRNA expression was significantly associated with early hepatic recurrence; thus, immunohistochemistry was performed to evaluate the expression levels of MAGEA10 protein in all 154 patients. Notably, a total of 
(a)

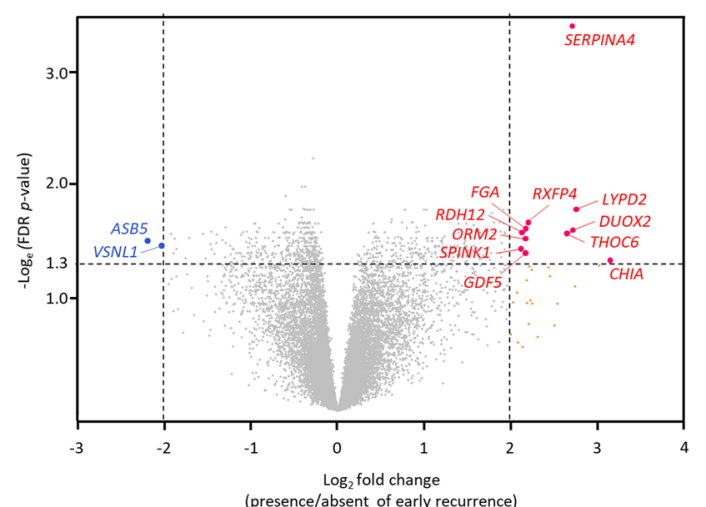

(c)

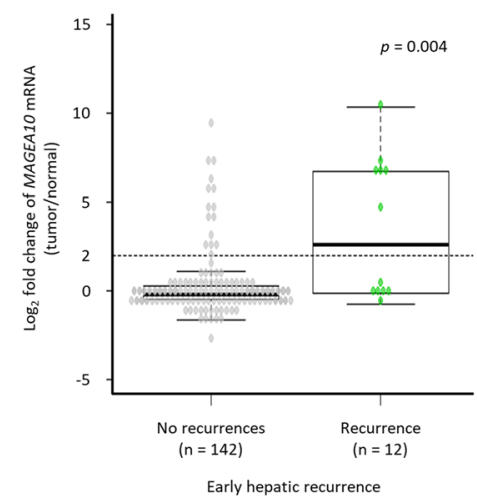

(b)

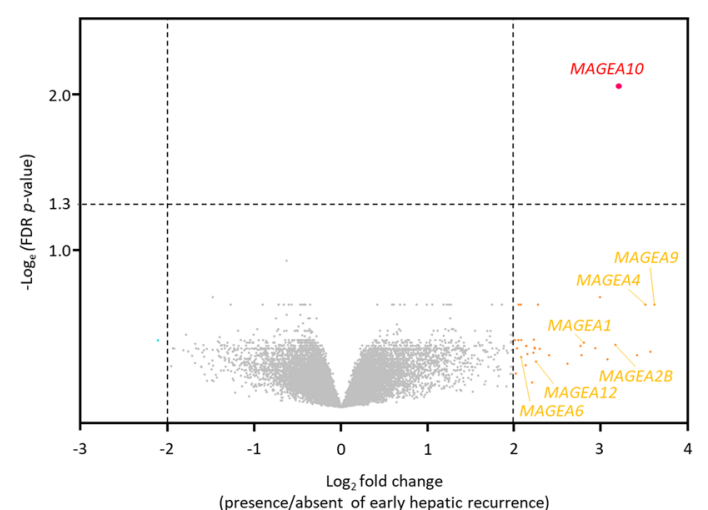

(d)

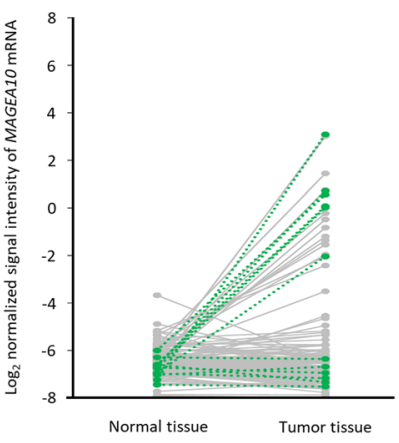

(e)

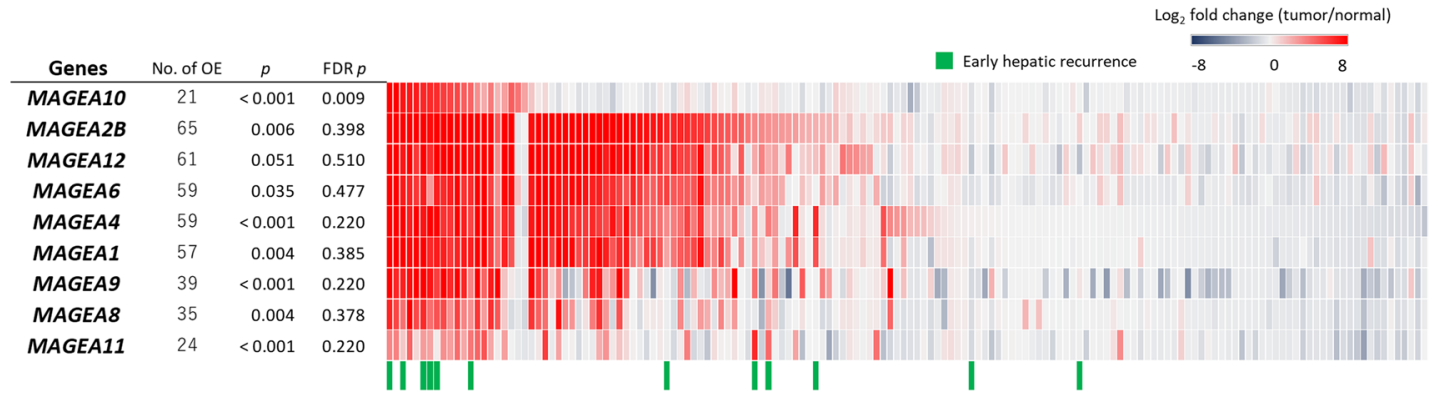

Fig. 2 Volcano plots for differentially expressed genes a between the presence and absence of early recurrence, and $\mathbf{b}$ between the presence and absence of early hepatic recurrence. c Association between MAGEA10 mRNA expression and early hepatic recurrence. A total of 6 in 12 patients with early hepatic recurrence show increased MAGEA10 mRNA expression ( $\geq$ fourfold change). d Comparison between MAGEA10 mRNA expression in normal tissue and tumor tissue. Recurrence is demonstrated by the green dotted line. e Gene expression profiling of MAGEA family genes in 154 gastric and gastroesophageal junction cancer patients. The heatmap represents the fold change of gene expression in tumor tissue compared to normal mucosal tissues. The number of OE indicates the number of patients with $a \geq$ fourfold increase in MAGEA10 mRNA expression. The $p$ value and FDR $p$ value show the relationship between early hepatic recurrence and expression of each gene 
(a)

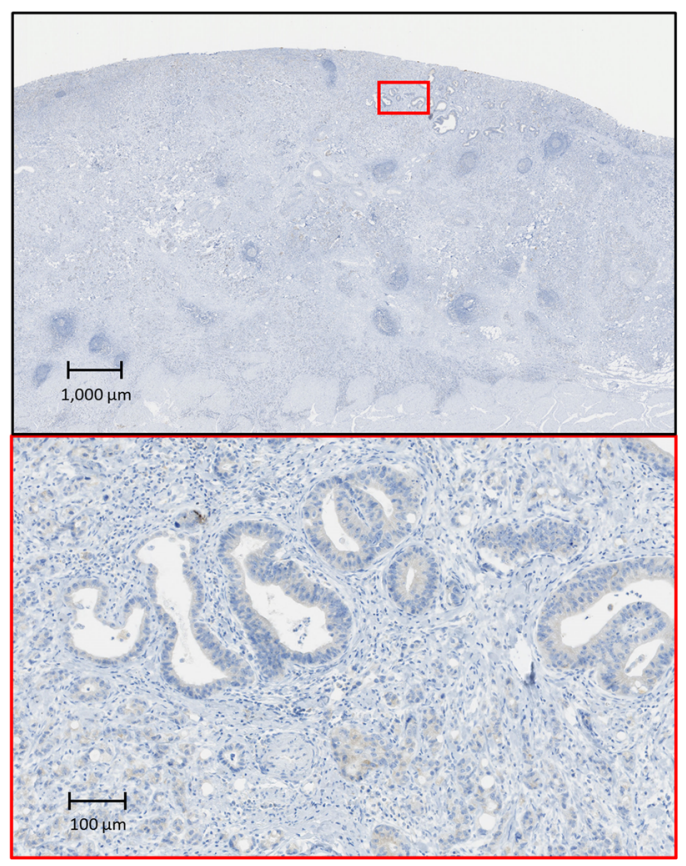

(b)

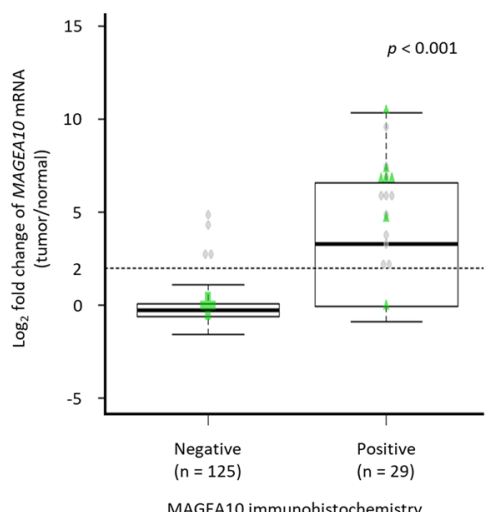

(d)

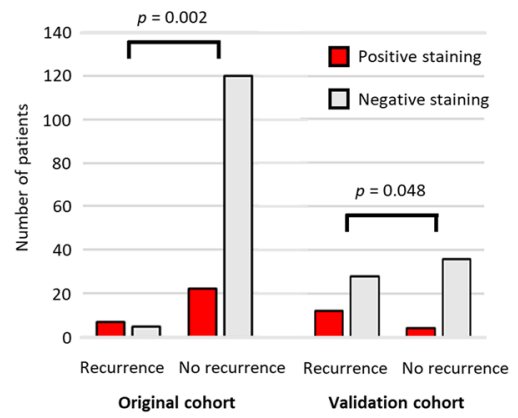

Positive staining for MAGEA10

Nuclear staining

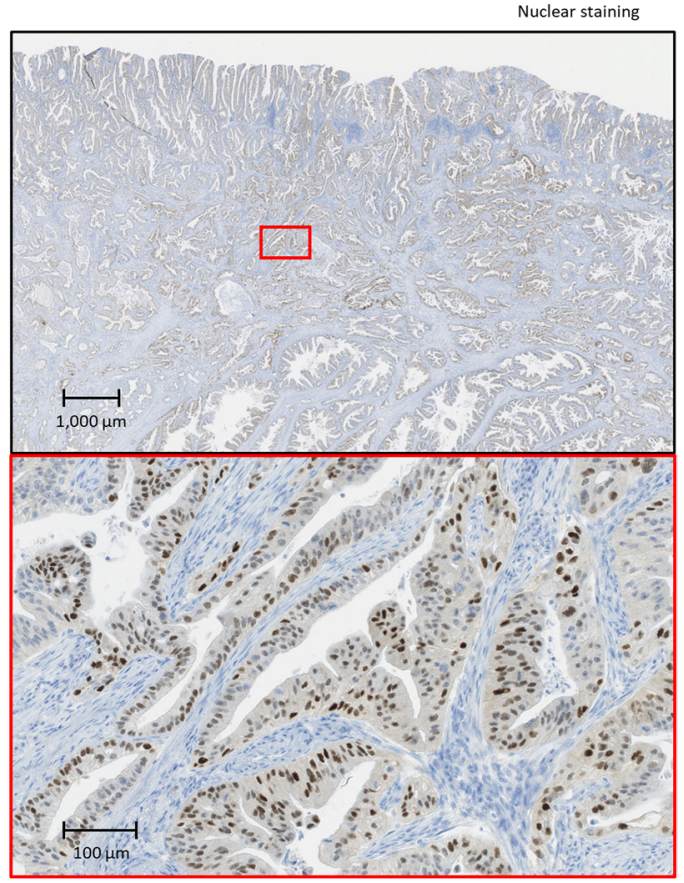

(c)

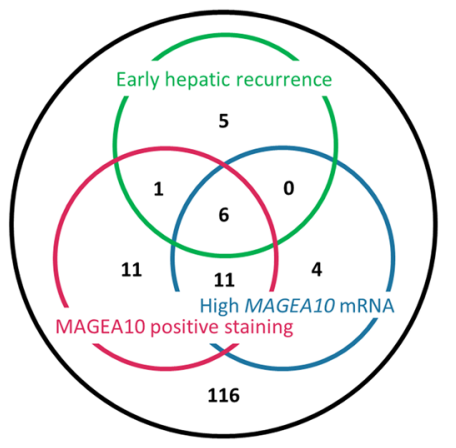


4Fig. 3 a Immunohistochemical evaluation for MAGEA10. Positive staining was defined as nuclear staining. b Correlation between protein expression on immunohistochemistry and mRNA expression in MAGEA10. Square indicates $\mathrm{f}$ samples with hepatic recurrence in negative staining; triangle indicates 7 samples with hepatic recurrence in positive staining; and the 21 samples with a greater than fourfold change are mainly illustrated by circles and triangles. c Distribution of patients with early hepatic recurrence, MAGEA10-positive staining, and high MAGEA10 mRNA expression. d Positive rate on immunohistochemistry of MAGEA10 in patients with and without early hepatic recurrence in original and validation cohorts

$29(18.8 \%)$ patients showed strong staining of MAGEA10, leading to an undeniable positive diagnosis (Fig. 3a). The distribution of positively stained cells showed a diffuse pattern in 13 patients, and a focal pattern in 16 patients. The histological types of the 29 patients with positive staining accounted for 20 differentiated type and 9 undifferentiated type (Table 2). Among them, all seven patients with early hepatic recurrence had tubular adenocarcinoma. The sensitivity and specificity of MAGEA10 staining for early hepatic recurrence were 58.3\% (95\% confidence interval [95\% CI] 27.7-84.8\%) and 84.5\% (95\% CI 77.5-90.0\%), respectively. Figure $3 \mathrm{~b}$ demonstrates significantly higher MAGEA10 mRNA expression in the 29 patients with positive staining compared to that in the 123 patients with negative staining. Figure $3 \mathrm{c}$ indicates the association between early hepatic recurrence, MAGEA10-positive staining, and high MAGEA10 mRNA expression. The details of 12 patients with early hepatic recurrence are described in Supplementary Table 3. The MAGEA10-positive staining rate in patients with and without early hepatic recurrence was $58.3 \%$ (95\% CI $27.7-84.8 \%$ ) and $15.5 \%$ (95\% CI $10.0-22.5 \%$ ) in the original cohort and $30.0 \%$ (95\% CI $16.6-46.5 \%$ ) and $10.0 \%$ (95\% CI 2.8-23.7\%) in the validation cohort, respectively. MAGEA10 staining was significantly associated with early hepatic recurrence in both cohorts (Fig. 3d).

\section{Consequence of immunohistochemistry for MAGEA10 on recurrence and survival after curative gastrectomy}

Positive staining for MAGEA10 was significantly associated with early hepatic recurrence $(p=0.002)$ (Table 2). Among the 21 patients with high MAGEA10 mRNA expression, early hepatic recurrence was found in $6(35.3 \%)$ of 17 patients with positive staining, but in none of the 4 patients with negative staining (Fig. 3b). There were 17 patients with high expression of both MAGEA10 mRNA and protein, and the specificity for early hepatic recurrence of double positive findings in high mRNA and protein expression was 91.9\%. During the entire follow-up period, hematogenous and peritoneal recurrences were significantly related to immunohistochemical findings; however, the other types of recurrences were not. Moreover, the cumulative incidence of early hepatic recurrence was higher in the group with positive MAGEA10 staining $(p<0.001)$ (Fig. $4 a)$. Reflecting the high incidence of hematogenous recurrence, relapsefree survival (RFS) was poorer in patients with positive MAGEA10 staining $(p=0.022)$, while overall survival (OS) was similar between the two groups $(p=0.967)$ (Fig. 4b, c). Survival analysis according to the MAGEA10 mRNA expression level ( $\geq$ four- or $<$ fourfold changes) showed similar findings in RFS $(p=0.020)$ and OS $(p=0.984)$ (Supplemental Fig. 1a, b).

\section{Discussion}

Comprehensive GEP analysis revealed that MAGEA10 is useful to predict early hepatic recurrence in gastric and gastroesophageal junction cancer. Genetic predictive markers for recurrence, including MAGEA10, will be important to improve survival. Intensive follow-up with imaging modality showed no survival benefit in any of the patients who underwent gastrectomy [23]. However, genomic approaches make it possible to refine patients at high risk for recurrence. Consequently, this may improve survival due to the use of aggressive adjuvant chemotherapy or close follow-up at-risk patients. It has been shown that the majority of recurrences of gastric cancer are found within 2 years after gastrectomy [24], and early recurrence is associated with poor survival [25]. Therefore, the present study focused on early recurrence within 2 year of surgery.

We failed to find significant mutated genes related to early recurrence in WES; however, we were able to discover candidate genes to predict recurrence using comprehensive GEP analysis. Although 13 genes were significantly regulated for early overall recurrence, only MAGEA10 upregulation was observed in early hepatic recurrence. In the previous studies, the expressions of TOP2A, GPR155, SYT5, and PRAME were significantly associated with hematogenous recurrence, including hepatic recurrence [11-14]. However, these genes were not selected as candidates for the prediction of early hepatic recurrence in this study.

Immunohistochemistry showed clear nuclear staining of MAGEA10, which was easily evaluated for positivity under the present staining conditions. The MAGEA10 protein is identified as an intranuclear protein with an apparent molecular weight of $70 \mathrm{kDa}$ [20]. MAGEA10 is not expressed in normal stomach tissue but is expressed in $14.9-52.1 \%$ of gastric cancers $[20,26]$. The protein expression of MAGEA10 is frequently observed in intestinal-type, but rarely in diffuse-type gastric cancers [20]; this histological difference in staining was similar to that observed in the present study. 
Table 2 Clinicopathological characteristics according to immunohistochemistry for MAGEA10

MAGEA10 immunohisto- $\quad p$ value chemistry

Positive Negative

$(n=29)(\%) \quad(n=125)(\%)$

\begin{tabular}{|c|c|c|c|}
\hline \multicolumn{3}{|l|}{ Age (years) } & \multirow[t]{3}{*}{0.892} \\
\hline Median & 76 & 71 & \\
\hline 25th-75th percentile & $76-83$ & $64-78$ & \\
\hline Sex & & & 0.820 \\
\hline Male & $20(69)$ & $90(72)$ & \\
\hline Female & $9(31)$ & $35(28)$ & \\
\hline Anatomical region & & & 0.217 \\
\hline Gastroesophageal junction & $6(21)$ & $14(11)$ & \\
\hline Gastric & $23(79)$ & $111(89)$ & \\
\hline Histology & & & 0.100 \\
\hline Differentiated type & $20(69)$ & $64(51)$ & \\
\hline Undifferentiated type & $9(31)$ & $61(49)$ & \\
\hline Primary tumor & & & 0.027 \\
\hline $\mathrm{T} 1$ & 0 & $4(3)$ & \\
\hline $\mathrm{T} 2$ & $8(28)$ & $13(10)$ & \\
\hline $\mathrm{T} 3$ & $12(41)$ & $39(31)$ & \\
\hline $\mathrm{T} 4$ & $9(31)$ & $69(55)$ & \\
\hline Regional lymph nodes & & & 0.295 \\
\hline No & $5(17)$ & $16(13)$ & \\
\hline N1 & $11(38)$ & $29(23)$ & \\
\hline $\mathrm{N} 2$ & $7(24)$ & $45(36)$ & \\
\hline N3 & $6(21)$ & $35(28)$ & \\
\hline Pathological stage & & & 0.034 \\
\hline II & $17(59)$ & $44(35)$ & \\
\hline III & $12(41)$ & $81(65)$ & \\
\hline Early hepatic recurrence & & & 0.002 \\
\hline Yes & $7(24)$ & $5(4)$ & \\
\hline No & $22(76)$ & $120(96)$ & \\
\hline Hematogenous recurrence & & & 0.001 \\
\hline Yes & $10(34)$ & $11(9)$ & \\
\hline No & $19(66)$ & $114(91)$ & \\
\hline Peritoneal recurrence & & & 0.025 \\
\hline Yes & 0 & $18(14)$ & \\
\hline No & $29(100)$ & $107(86)$ & \\
\hline Distant lymph node recurrence & & & 0.400 \\
\hline Yes & $3(10)$ & $7(6)$ & \\
\hline No & $26(90)$ & $118(94)$ & \\
\hline Locoregional recurrence & & & 0.091 \\
\hline Yes & $2(7)$ & $1(1)$ & \\
\hline No & $27(93)$ & $124(99)$ & \\
\hline
\end{tabular}

MAGEA family genes are located in Xq28 and are frequently coexpressed in cancer [27, 28]. The present study showed coexpression between MAGEA10 and other MAGEA family genes that are overexpressed in various
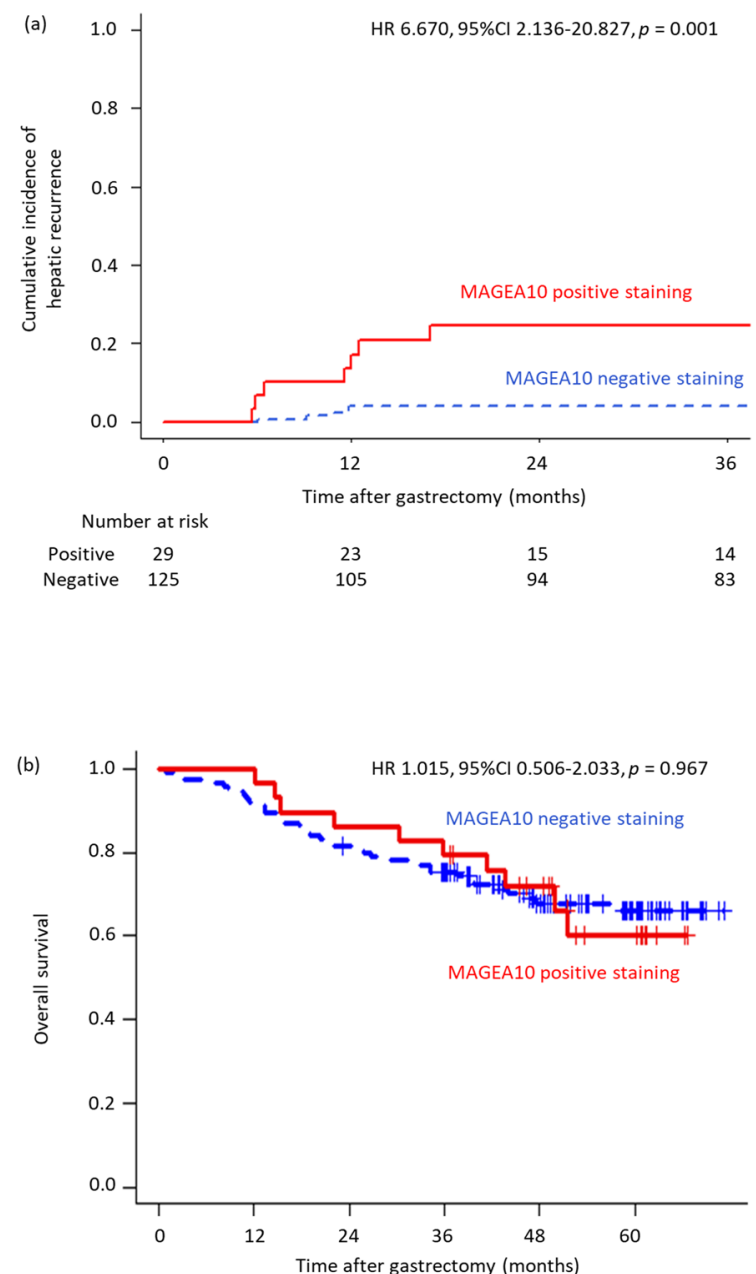

Number at risk $\begin{array}{ccccccc}\text { Positive } & 29 & 29 & 25 & 23 & 17 & 8 \\ \text { Negative } & 125 & 115 & 101 & 89 & 54 & 26\end{array}$

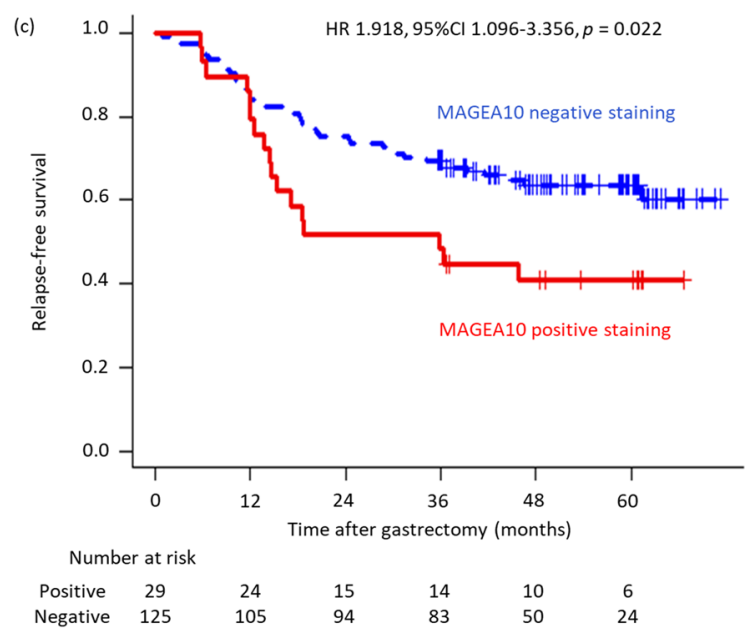

Fig. 4 Early hepatic recurrence and survival analysis according to immunohistochemical evaluation of MAGEA10. a Cumulative incidence of early hepatic recurrence. b Overall survival. c Relapse-free survival 
cancers and are known as tumor associated antigens [29]. Upregulation of MAGE family genes triggers ubiquitination and degradation of multiple tumor suppressors, such as TP53, AMPK $\alpha 1$, and ZNF382. Moreover, MAGEA proteins directly interact with TP53 and occlude the binding of TP53 to TP53-responsive promoters, leading to decreased TP53-dependent transcription, cell cycle arrest, and apoptosis [30]. Overexpression of MAGEA family genes is observed in liver metastases of gastric [26], colorectal [31, 32], and pancreatic cancer [33]. The mechanism leading to liver metastases is considered to be the inhibition of TP53 transcription activity and the increase in TP53 degradation [34]. In gastric cancer, MAGEA10 was more highly expressed in liver metastases than in the primary site [26].

A previous report showed that overexpression of MAGEA6 is related to overall recurrence, but not each pattern of recurrence [35]. Furthermore, many studies have revealed that MAGEA protein expression, such as MAGEA1, A3, A6, A10, and A12, correlated with lymph node metastasis, advanced stage of cancer, and worse survival in gastric cancer [26, 35-39]. The present study showed a high incidence of hepatic recurrence and poor RFS in patients with positive MAGEA10 staining. Moreover, specificity was increased in the combination of protein and mRNA expression in MAGEA10, which supports the use of MAGEA10 as a key biomarker for hepatic recurrence. OS was almost equal irrespective of staining. In contrast to a previous study including patients possessing synchronous liver metastasis, MAGEA10 is not a prognostic marker in patients who undergo curative gastrectomy for stage II or III gastric cancer [26]. A low proportion of peritoneal recurrences in patients with positive MAGEA10 staining and recent therapeutic advances in surgery and chemotherapy for liver metastases may cause this discordance. Compared to peritoneal recurrence, gastric cancer with liver metastases has a relatively longer survival $[6,7]$.

Immunotherapy targeting MAGEA family genes has gained increasing attention in recent years because these genes are highly expressed in many types of cancers. However, the efficacy of MAGEA3 cancer immunotherapy was not proven in a Phase 3 trial for lung cancer and melanoma $[40,41]$. However, novel strategies of tumor vaccines in combination with immune-checkpoint inhibitors are currently in progress. Thus, MAGEA10 may be a good candidate for future immunotherapy in advanced gastric cancer.

This study has several limitations. First, this was a single institutional study and the number of patients with early hepatic recurrence was small. Second, immunohistochemistry was not performed for other genes in the MAGEA family. Although immunohistochemistry analysis of MAGEA10 demonstrated high specificity for predicting early hepatic recurrence, the sensitivity was not enough. Therefore, using a combination approach, including the anti-pan-MAGEA antibody, may improve prediction sensitivity. Third, the follow-up period was relatively short, which meant that the OS and RFS analysis was not robust.

\section{Conclusions}

MAGEA10 is a good predictive biomarker for early hepatic recurrence after curative gastrectomy for advanced gastric and gastroesophageal junction cancer. This marker is valuable to select patients who require intensive follow-up after surgery, since early hepatic recurrence has a possibility to be cured by resection.

Acknowledgements We thank Mr. Koji Muramatsu for his valuable technical pathological assistance.

Author contributions KF and MT: designed the study; KU and KY: developed the concept of the project HOPE; KF, KO, MS, KH, KU, and YA: performed the experiments; TN: analyzed the data; DA and TS: evaluated pathological specimens; KF: wrote the paper, with contributions from MT KO, KN, KH, and $\mathrm{YK}$.

Funding This research was supported in part by the Shizuoka Prefectural Government, Japan.

\section{Compliance with ethical standards}

Conflict of interest Dr. Terashima reports personal fees from Taiho, personal fees from Chugai, personal fees from Ono, personal fees from BMS, personal fees from Yakult, personal fees from Takeda, personal fees from Eli Lilly, personal fees from Pfizer, and personal fees from Daiichi Sankyo, outside the submitted work. The other authors have nothing to disclose.

Ethical approval All procedures were conducted in accordance with the ethical standards of the corresponding committees on human experimentation (institutional and national) and with the Helsinki Declaration of 1964 and later versions.

Informed consent Informed consent was obtained from all patients.

\section{References}

1. Yoshikawa T, Terashima M, Mizusawa J, Nunobe S, Nishida Y, Yamada T, et al. Four courses versus eight courses of adjuvant S-1 for patients with stage II gastric cancer (JCOG1104 [OPAS-1]): an open-label, phase 3, non-inferiority, randomised trial. Lancet Gastroenterol Hepatol. 2019;4:208-16.

2. Yoshida K, Kodera Y, Kochi M, Ichikawa W, Kakeji Y, Sano T, et al. Addition of docetaxel to oral fluoropyrimidine improves efficacy in patients with stage III gastric cancer: interim analysis of JACCRO GC-07, a randomized controlled trial. J Clin Oncol. 2019;37:1296-304. 
3. Japanese Gastric Cancer A. Japanese gastric cancer treatment guidelines 2018 (5th edition). Gastric Cancer. 2020. (Epub ahead of print).

4. Fujiya K, Tokunaga M, Makuuchi R, Nishiwaki N, Omori H, Takagi W, et al. Early detection of nonperitoneal recurrence may contribute to survival benefit after curative gastrectomy for gastric cancer. Gastric Cancer. 2017;20:141-9.

5. Kinoshita T, Kinoshita T, Saiura A, Esaki M, Sakamoto H, Yamanaka T. Multicentre analysis of long-term outcome after surgical resection for gastric cancer liver metastases. Br J Surg. 2015;102:102-7.

6. Markar SR, Mackenzie H, Mikhail S, Mughal M, Preston SR, Maynard ND, et al. Surgical resection of hepatic metastases from gastric cancer: outcomes from national series in England. Gastric Cancer. 2017;20:379-86.

7. Markar S, Mikhail S, Malietzis G, Athanasiou T, Mariette C, Sasako M, et al. Influence of surgical resection of hepatic metastases from gastric adenocarcinoma on long-term survival: systematic review and pooled analysis. Ann Surg. 2016;263:1092-101.

8. Yoshikawa K, Kitaoka H. Clinicopathologic studies of gastric cancer with metastasis to the liver-based on the cases detected at initial surgery. Jpn J Clin Oncol. 1984;14:81-6.

9. Fang W-L, Huang K-H, Lan Y-T, Lin C-H, Chang S-C, Chen $\mathrm{M}-\mathrm{H}$, et al. Mutations in PI3K/AKT pathway genes and amplifications of PIK3CA are associated with patterns of recurrence in gastric cancers. Oncotarget. 2016;7:6201-20.

10. Ikari N, Serizawa A, Mitani S, Yamamoto M, Furukawa T. Near-comprehensive resequencing of cancer-associated genes in surgically resected metastatic liver tumors of gastric cancer. Am J Pathol. 2019;189:784-96.

11. Terashima M, Ichikawa W, Ochiai A, Kitada K, Kurahashi I, Sakuramoto S, et al. TOP2A, GGH, and PECAM1 are associated with hematogenous, lymph node, and peritoneal recurrence in stage II/III gastric cancer patients enrolled in the ACTS-GC study. Oncotarget. 2017;8:57574-822.

12. Shimizu D, Kanda M, Tanaka H, Kobayashi D, Tanaka C, Hayashi M, et al. GPR155 serves as a predictive biomarker for hematogenous metastasis in patients with gastric cancer. Sci Rep. 2017;7:42089.

13. Kanda M, Tanaka H, Shimizu D, Miwa T, Umeda S, Tanaka C, et al. SYT7 acts as a driver of hepatic metastasis formation of gastric cancer cells. Oncogene. 2018;37:5355-66.

14. Baba H, Kanda M, Sawaki K, Umeda S, Miwa T, Shimizu D, et al. PRAME as a potential biomarker for liver metastasis of gastric cancer. Ann Surg Oncol. 2020;27:2071-80.

15. Yamaguchi K, Urakami K, Ohshima K, Mochizuki T, Akiyama $\mathrm{Y}$, Uesaka $\mathrm{K}$, et al. Implementation of individualized medicine for cancer patients by multiomics-based analyses-the Project HOPE. Biomed Res. 2014;35:407-12.

16. Shimoda Y, Nagashima T, Urakami K, Tanabe T, Saito J, Naruoka A, et al. Integrated next-generation sequencing analysis of whole exome and 409 cancer-related genes. Biomed Res. 2016;37:367-79.

17. Nagashima T, Shimoda Y, Tanabe T, Naruoka A, Saito J, Serizawa M, et al. Optimizing an ion semiconductor sequencing data analysis method to identify somatic mutations in the genomes of cancer cells in clinical tissue samples. Biomed Res. 2016;37:359-66.

18. Nagashima T, Yamaguchi K, Urakami K, Shimoda Y, Ohshima K, Tanabe T, et al. Japanese version of cancer genome atlas, JCGA, analyzed by fresh frozen tumors obtained from 5143 cancer patients. Cancer Sci. 2019. (Epub ahead of print)

19. Ohshima K, Hatakeyama K, Nagashima T, Watanabe Y, Kanto $\mathrm{K}$, Doi $\mathrm{Y}$, et al. Integrated analysis of gene expression and copy number identified potential cancer driver genes with amplification-dependent overexpression in 1,454 solid tumors. Sci Rep. 2017;7:641

20. Schultz Thater E, Piscuoglio S, Iezzi G, Le Magnen C, Zajac $\mathrm{P}$, Carafa V, et al. MAGE-A10 is a nuclear protein frequently expressed in high percentages of tumor cells in lung, skin and urothelial malignancies. Int J Cancer. 2011;129:1137-48.

21. Cancer Genome Atlas Research N. Comprehensive molecular characterization of gastric adenocarcinoma. Nature. 2014;513:202-9.

22. Ichikawa H, Nagahashi M, Shimada Y, Hanyu T, Ishikawa T, Kameyama H, et al. Actionable gene-based classification toward precision medicine in gastric cancer. Genome Med. 2017;9:93.

23. Bjerring OS, Fristrup CW, Pfeiffer P, Lundell L, Mortensen MB. Phase II randomized clinical trial of endosonography and PET/ CT versus clinical assessment only for follow-up after surgery for upper gastrointestinal cancer (EUFURO study). Br J Surg. 2019; 106:1761-8.

24. Feng XY, Chen YB, Wang W, Guan YX, Li YF, Chen S, et al. Time-varying pattern of recurrence risk for gastric cancer patients. Med Oncol. 2013;30:514.

25. Mitani S, Kadowaki S, Hasegawa H, Wakatsuki T, Hara H, Tajika M, et al. Systemic chemotherapy for gastric cancer with early recurrence after adjuvant S-1 monotherapy: a multicenter retrospective study. Int J Clin Oncol. 2019;24:1197-203.

26. Suzuki S, Sasajima K, Sato Y, Watanabe H, Matsutani T, Iida $\mathrm{S}$, et al. MAGE-A protein and MAGE-A10 gene expressions in liver metastasis in patients with stomach cancer. Br J Cancer. 2008;99:350-6.

27. Ries J, Schultze Mosgau S, Neukam F, Diebel E, Wiltfang J. Investigation of the expression of melanoma antigen-encoding genes (MAGE-A1 to -A6) in oral squamous cell carcinomas to determine potential targets for gene-based cancer immunotherapy. Int J Oncol. 2005;26:817-24.

28. Cuffel C, Rivals JP, Zaugg Y, Salvi S, Seelentag W, Speiser $\mathrm{DE}$, et al. Pattern and clinical significance of cancer-testis gene expression in head and neck squamous cell carcinoma. Int J Cancer. 2011;128:2625-34.

29. Lian Y, Meng L, Ding P, Sang M. Epigenetic regulation of MAGE family in human cancer progression-DNA methylation, histone modification, and non-coding RNAs. Clin Epigenet. 2018;10:115.

30. Marcar L, Maclaine NJ, Hupp TR, Meek DW. Mage-A cancer/ testis antigens inhibit $\mathrm{p} 53$ function by blocking its interaction with chromatin. Cancer Res. 2010;70:10362-70.

31. Mori M, Inoue H, Mimori K, Shibuta K, Baba K, Nakashima H, et al. Expression of MAGE genes in human colorectal carcinoma. Ann Surg. 1996;224:183-8.

32. Hasegawa H, Mori $M$, Haraguchi $M$, Ueo $H$, Sugimachi $K$, Akiyoshi T. Expression spectrum of melanoma antigen-encoding gene family members in colorectal carcinoma. Arch Pathol Lab Med. 1998;122:551-4.

33. Bert T, Lubomierski N, Gangsauge S, Munch K, Printz H, Prasnikar N, et al. Expression spectrum and methylation-dependent regulation of melanoma antigen-encoding gene family members in pancreatic cancer cells. Pancreatology. 2002;2:146-54.

34. Meng L, Liu F, Ju Y, Ding P, Liu S, Chang S, et al. Tumor suppressive miR-6775-3p inhibits ESCC progression through forming a positive feedback loop with p53 via MAGE-A family proteins. Cell Death Dis. 2018;9:1057.

35. Endo M, Kanda M, Sawaki K, Shimizu D, Tanaka C, Kobayashi $\mathrm{D}$, et al. Tissue expression of melanoma-associated antigen A6 and clinical characteristics of gastric cancer. Anticancer Res. 2019;39:5903-10.

36. Jung EJ, Kim MA, Lee HS, Yang HK, Lee YM, Lee BL, et al. Expression of family A melanoma antigen in human gastric carcinoma. Anticancer Res. 2005;25:2105-11. 
37. Ogata K, Aihara R, Mochiki E, Ogawa A, Yanai M, Toyomasu $\mathrm{Y}$, et al. Clinical significance of melanoma antigen-encoding gene-1 (MAGE-1) expression and its correlation with poor prognosis in differentiated advanced gastric cancer. Ann Surg Oncol. 2011;18:1195-203.

38. Wu J, Wang J, Shen W. Identification of MAGEA12 as a prognostic outlier gene in gastric cancers. Neoplasma. 2017;64:238-43.

39. Futawatari N, Fukuyama T, Yamamura R, Shida A, Takahashi Y, Nishi Y, et al. Early gastric cancer frequently has high expression of KK-LC-1, a cancer-testis antigen. World J Gastroenterol. 2017;23:8200-6.

40. Vansteenkiste J, Cho B, Vanakesa T, De Pas T, Zielinski M, Kim $\mathrm{M}$, et al. Efficacy of the MAGE-A3 cancer immunotherapeutic as adjuvant therapy in patients with resected MAGE-A3-positive non-small-cell lung cancer (MAGRIT): a randomised, double-blind, placebo-controlled, phase 3 trial. Lancet Oncol. 2016;17:822-35.

41. Dreno B, Thompson J, Smithers B, Santinami M, Jouary T, Gutzmer R, et al. MAGE-A3 immunotherapeutic as adjuvant therapy for patients with resected, MAGE-A3-positive, stage III melanoma (DERMA): a double-blind, randomised, placebocontrolled, phase 3 trial. Lancet Oncol. 2018;19:916-29.

Publisher's Note Springer Nature remains neutral with regard to jurisdictional claims in published maps and institutional affiliations. 